\title{
Rizicultures asiatiques : Permanence d'une céréale essentielle sur un continent en mutation
}

Guy Trébuil ${ }^{1}$

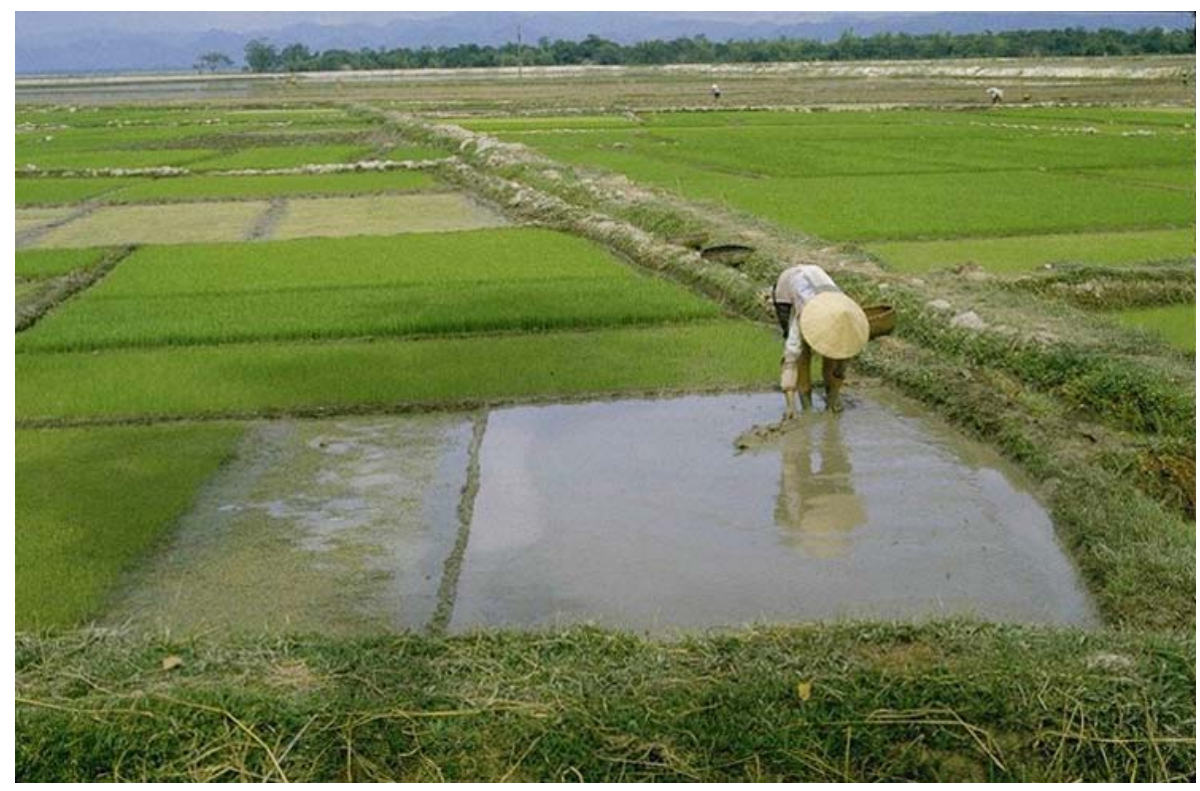

Riz irrigué - Planage manuel d'une minuscule pépinière au cœur du delta du Fleuve rouge, nord du Vietnam (@ Juillet 1986 / G. Trébuil)

Plante domestiquée en Asie il y a environ 8.000 à 10.000 ans, le riz (espèce Oryza sativa) constitue toujours au début du XXI ${ }^{\text {ème }}$ siècle la première céréale de l'alimentation humaine et la principale source d'énergie et d'une part significative des protéines pour quelques trois milliards d'hommes. Si sa consommation atteint jusqu'à $200 \mathrm{~kg}$ de riz blanc par personne et par an dans certains pays en développement sur ce continent, elle n'est plus que d'environ 50 kg/tête/an de grain, mais de haute qualité ceux-là, dans les pays les plus riches comme le Japon. La production mondiale annuelle de riz dépasse 600 millions de tonnes de paddy (grain non décortiqué) et demeure située à $90 \%$ en Asie où les grands pays producteurs sont toujours la Chine, l’Inde, l’Indonésie, le Bangladesh, le Vietnam, la Thaïlande.

La production cruciale de ce "grain de vie " repose encore sur une myriade de minuscules exploitations agricoles familiales, disposant très souvent de moins d'un hectare de rizière par ménage, par exemple en Chine, et d'environ 4 ha en moyenne en Thaïlande bien mieux dotée en terres agricoles. Les rizicultures occupent environ 150 millions d'hectares de par le monde, soit $15 \%$ de la superficie cultivée et près d'un quart du total des surfaces en grains. Leur mise en œuvre mobilise plus de 200 millions de riziculteurs, parmi lesquels la part des pluriactifs augmente rapidement sur les traces du précédent japonais. Il s’agit donc de la plus importante activité humaine à la surface du globe !

${ }^{1}$ Géo-agronome, unité de recherche gestion des ressources renouvelables et environnement (Green), département Environnements et Sociétés, Cirad, Montpellier. Auteur avec M. Hossain de l’ouvrage «Le Riz: enjeux écologiques et économiques» publié en 2004 dans la collection Mappemonde aux Éditions Belin, Paris. 


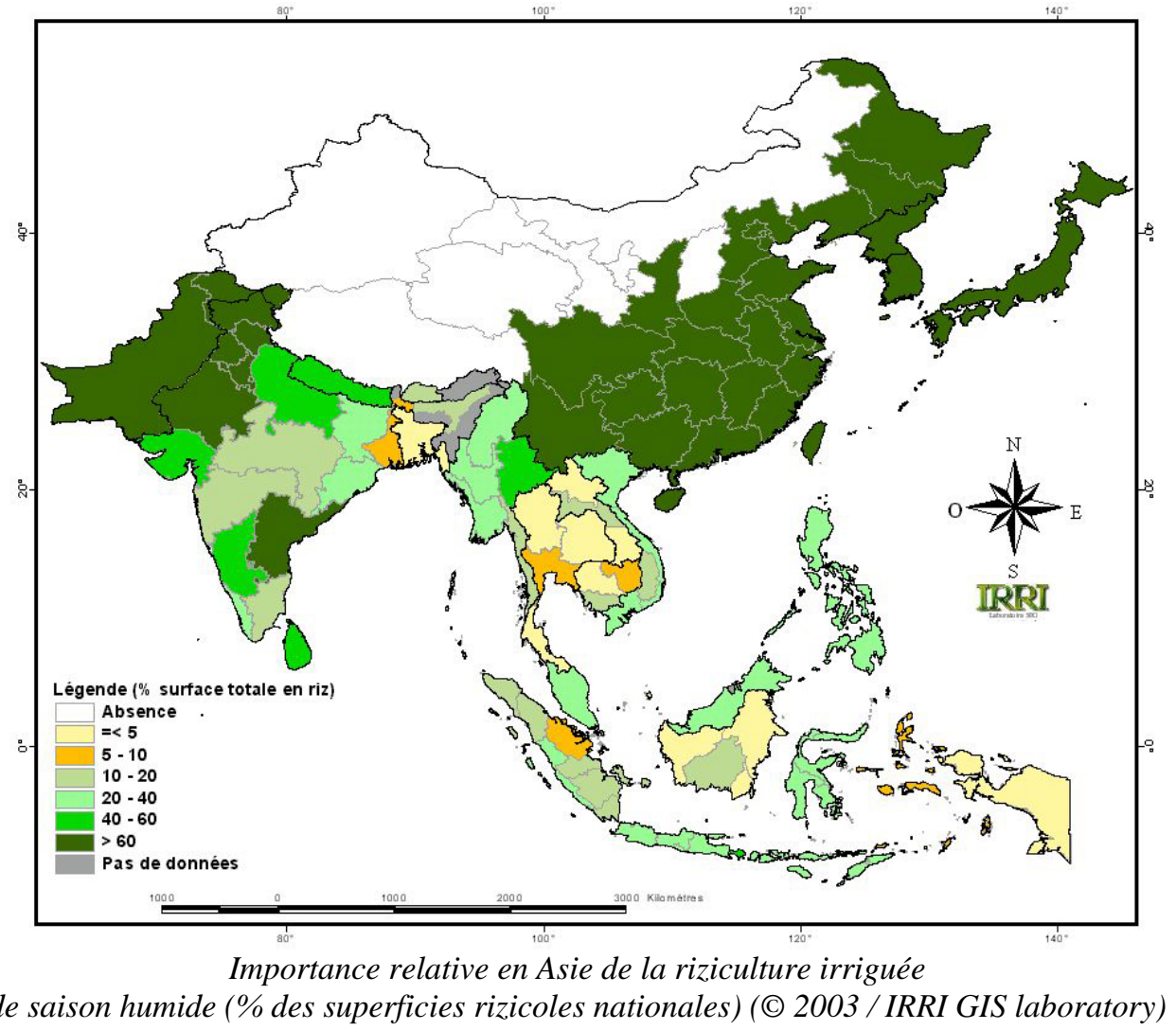

Le riz produit est très largement autoconsommé par les ménages rizicoles qui écoulent leurs surplus sur les marchés locaux et nationaux. Bon mal an, seulement 6 à $7 \%$ de la récolte sont échangés sur le marché mondial, les principaux exportateurs étant la Thaïlande, le Vietnam, les Etats-Unis, l'Inde et le Pakistan. Si les pays industrialisés importent quelques millions de tonnes de riz aromatiques, basmatis ou thaïs, à haute valeur commerciale, des volumes bien plus importants sont écoulés dans le monde tropical en développement, notamment en Afrique sub-saharienne et au moyen orient. Activité humaine hautement identitaire et profondément enracinée dans les cultures asiatiques qu'elles contribuent à rapprocher (voir la gerbe de riz du logo de l'ASEAN ${ }^{2}$ ), il est très fréquent que la petite production familiale des différents socio-écosystèmes rizicoles se perpétue en dehors de tout objectif de rentabilité économique. Si l'empereur du Japon demeure un riziculteur sacré, les semailles royales de début mai continuent de lancer le démarrage du cycle rizicole de mousson humide à travers la Thaïlande. C'est un fait remarquable dans cette partie du monde, abonnée aux taux de croissance à deux chiffres lors des dernières décennies, mais même là où aujourd'hui « les rizières sont sous le béton [...] le riz a laissé son empreinte sur nous » écrit S. Rushdie.

\footnotetext{
${ }^{2}$ Association des nations d'Asie du Sud-Est.
} 


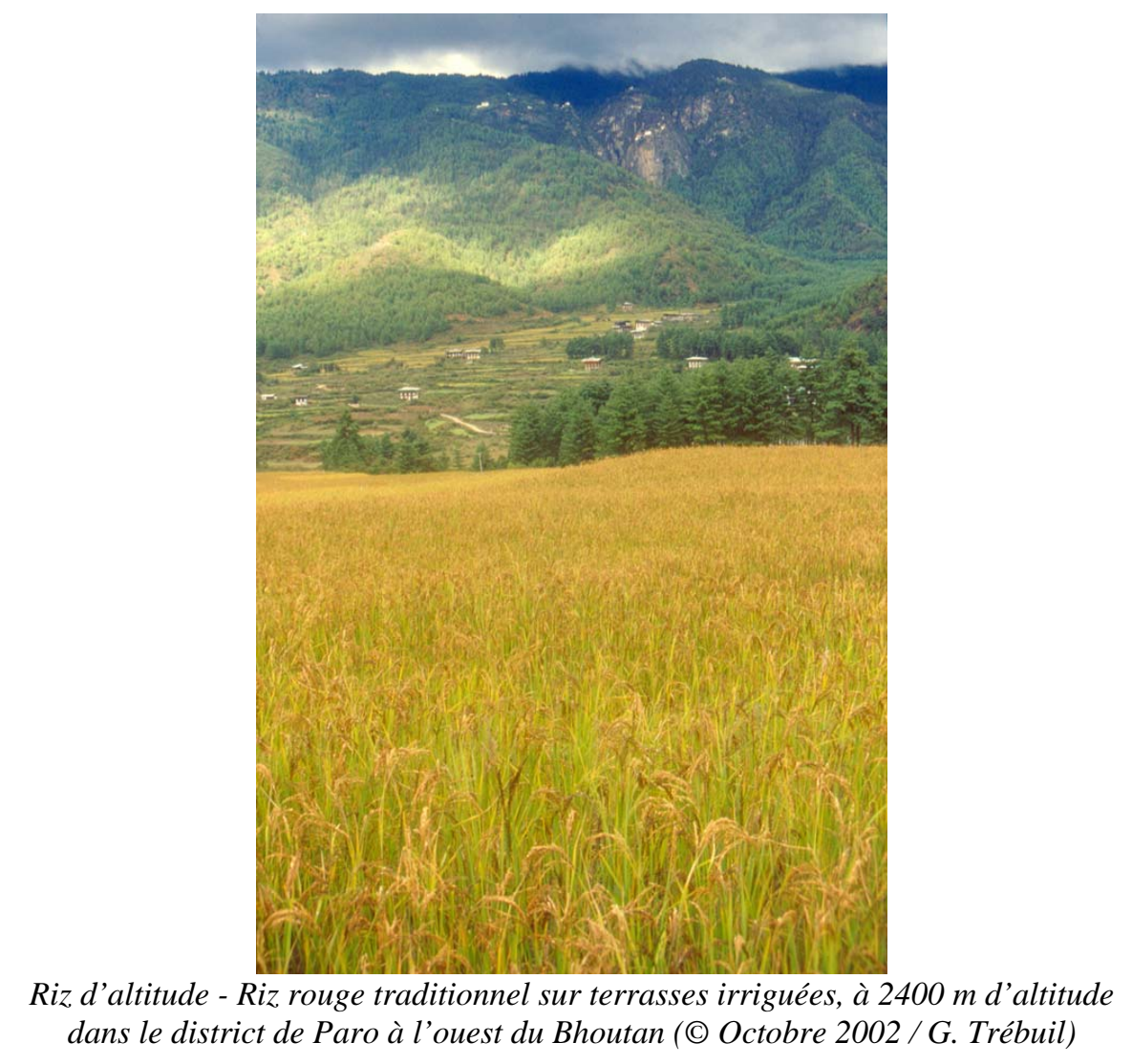

Cette céréale essentielle est produite dans des écologies extrêmement variées depuis les grandes plaines côtières jusqu'aux terrasses montagnardes à 2700 mètres d'altitude dans l'Himalaya, et de l'équateur jusqu'à 53 degrés de latitude Nord dans la province septentrionale chinoise du Heilongjiang. L'écosystème à riz irrigué, où deux ou trois cycles culturaux peuvent se succéder par an sur le même casier, est caractérisé par un meilleur contrôle de l'eau en surface, le meilleur herbicide ici. Avec plus de la moitié des surfaces rizicoles et les trois quarts de la production totale, c'est le domaine des grands «bols de riz » nourriciers des deltas et plaines alluviales densément peuplés (plus de 10 habitants par hectare de rizière) qui domine largement en Asie orientale, où la Chine nourrit plus du cinquième de la population mondiale avec $9 \%$ des terres arables. Si le rendement moyen en paddy y sont de 5 à 6 tonnes/ha/cycle cultural en saison humide, il peut grimper jusqu'à 10 voire12 t/ha en saison sèche à nébulosité réduite, notamment dans les rizières chinoises plantées en « super hybrides » précoces synthétisant plus de $100 \mathrm{~kg}$ de paddy par hectare et par jour ! C’est en riziculture irriguée que la révolution verte rizicole (1965-1990), en combinant innovations technologiques (variétés productives semi-naines répondant à l’azote) et politiques incitatrices (infrastructures hydrauliques, prix et marchés, recherche et vulgarisation), a permis un triplement de la productivité physique du sol, la mécanisation intermédiaire des opérations culturales (le petit motoculteur multi-usages ubiquiste y a joué un rôle clef), l'augmentation de l'emploi agricole, la baisse tendancielle du prix du riz qui a grandement facilité le décollage industriel, mais aussi la montée des atteintes à l'intégrité du milieu (eau, pesticides, fin de la pisciculture en rizière, etc.). Ces dégradations doivent être limitées au moyen de nouveaux systèmes de culture productifs mais reposant beaucoup plus qu'auparavant sur les régulations écologiques que sur une artificialisation encore plus poussée du milieu rizicole. Le challenge est impressionnant, car ces systèmes irrigués devront nourrir un nombre croissant de consommateurs avec moins de terres (urbanisation, salinisation des sols, diversification agricole), moins de bras (industrialisation et services absorbant la main d'œuvre rurale), 
moins d'intrants chimiques (engrais minéraux plus coûteux et arrêt de la surconsommation de pesticides) et moins d'eau agricole (compétition croissante avec les autres usages de cette ressource). Car produire un kilogramme de paddy avec jusqu'à cinq mètres cubes d'eau, et en relâchant dans le milieu les deux tiers de l'azote apporté, ne sera plus possible dans la plupart des régions!

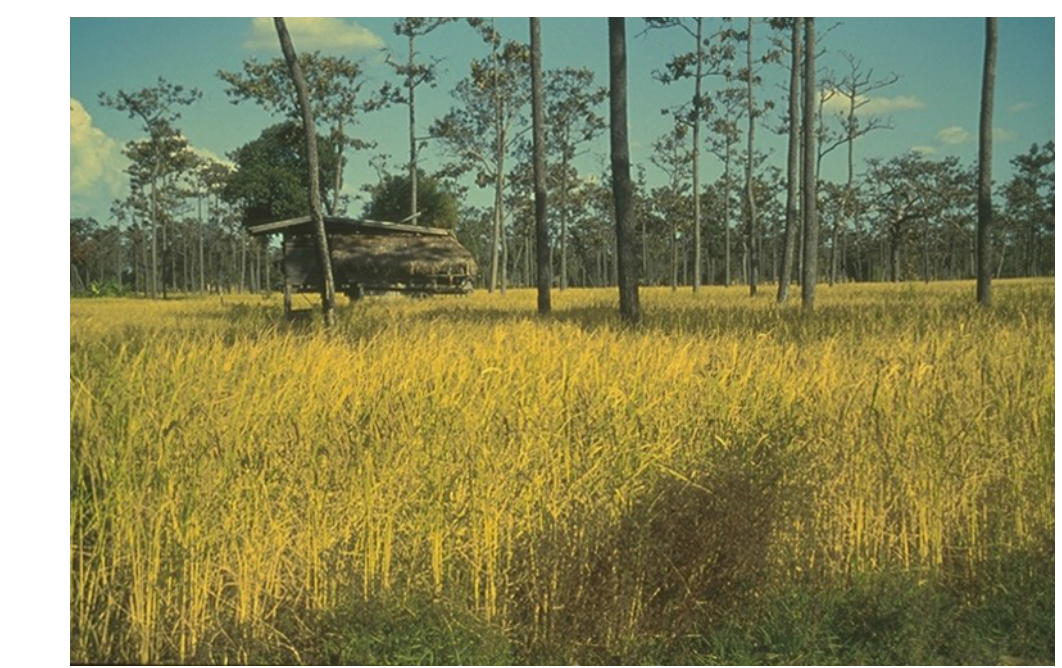

Riz inondé - Parcelles de riz traditionnel aromatique « à odeur de jasmin » à Ubon Ratchathani au nord-est de la Thaïlande (C Novembre 1994 / G. Trébuil)

Avec un quart des surfaces, la riziculture inondée en casiers également endigués et étagés, mais dont la submersion dépend de la pluviométrie en l'absence d'apport d'eau par irrigation, ne permet pas le plus souvent un contrôle satisfaisant de l'hydrologie de surface. Pas de double ou triple culture annuelle, mais ce système demeure très répandu en Asie du Sud et du Sud-Est où il est sujet aux déficits hydriques en début et fin de cycle, ainsi qu'aux inondations profondes lors du pic de la mousson humide. Ignorée par la révolution verte, source de fortes disparités interrégionales, le manque de maîtrise de l'eau ne permet pas l'adoption de variétés semi-naines productives, et les rendements de ses cultivars rustiques restent de l'ordre de 2 à 3 t/ha. La riziculture d'eau profonde, côtière ou à riz flottants (aux tiges pouvant dépasser 5 mètres de longueur) constitue un écosystème plus marginal, en contraction, et à faible productivité physique (rendements de l'ordre de 1-1,5 t de paddy/ha), souvent converti en fermes aquacoles (poissons, crevettes, crabes, etc.). Il en est de même de la riziculture pluviale, non endiguée et sans submersion des parcelles, souvent pratiquée sur les hautes terres en pente au moyen de l'abattis-brûlis. Au fil de l'intégration de ces régions plus reculées au marché, et face à la pression croissante de conservation de la couverture forestière, elle laisse largement la place à une palette diversifiée de cultures commerciales (maïs, fruits et légumes, hévéa, etc.). 


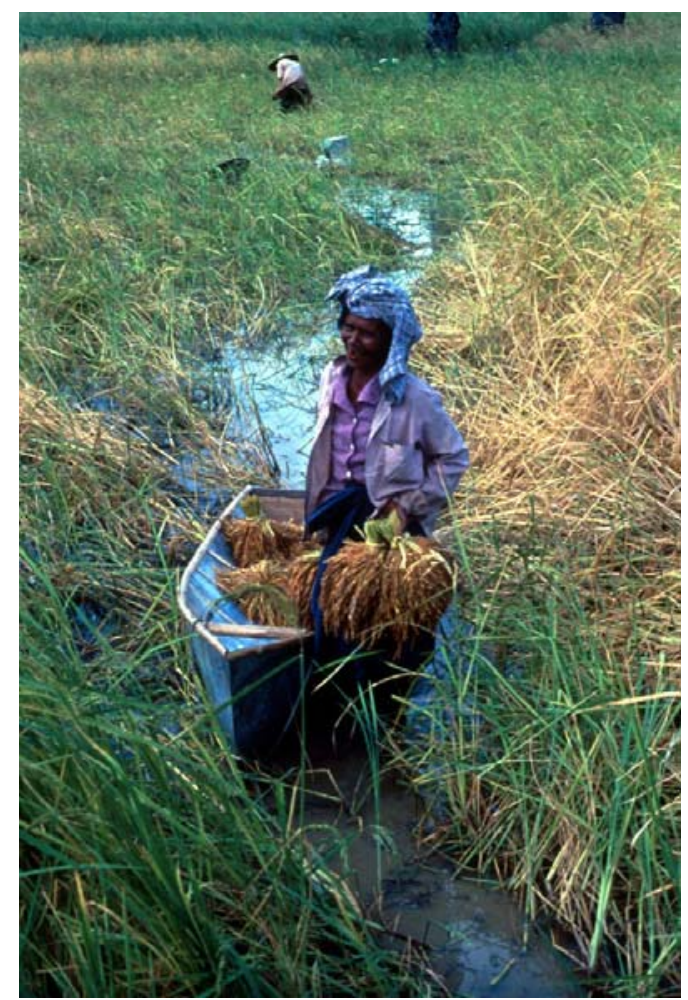

Rizière à submersion profonde - Récolte panicule par panicule de riz traditionnels très tardifs dans une dépression inondée au sud de la Thaïlande ( Février 1983 / G. Trébuil)

Le riz était déjà cultivé dès le néolithique supérieur dans la moyenne vallée du Yangtse (où la présence d'un système d'irrigation et de drainage de 7000 ans a été mise à jour à Chengtoushan) ainsi que la haute vallée du fleuve Huai en Chine méridionale, puis un peu plus tard dans l'Uttar Pradesh au nord-est de l'Inde. Les autres plantes alimentaires de l'époque pouvant être l'orge, le millet, le sarrasin ou des tubercules. Les sélectionneurs admettent qu'Oryza sativa a été domestiquée, de manière indépendante en Chine et en Inde, à partir de formes annuelles de l'espèce sauvage O. rufipogon en donnant naissance aux deux principaux groupes, indicas et japonicas, clairement distingués par leur origine géographique, leurs traits morphologiques et agronomiques, ainsi que par les marqueurs biochimiques et moléculaires, ce qui rend leur recombinaison difficile ${ }^{3}$. Des travaux récents montrent que les génomes indicas et japonicas auraient divergé bien avant cette domestication, il y a au moins 200.000 ans et probablement 2 ou 3 millions d'années avant, sans doute en lien avec la surrection de la barrière himalayenne. Si la sous-espèce indica est largement répandue dans les rizicultures tropicales irriguée ou inondée, les japonicas sont plutôt utilisés en riziculture irriguée tempérée et d'altitude ainsi qu'en régime pluvial. En Chine, la culture des fonds de vallées et des deltas avec mise en boue et repiquage de la rizière aurait précédé la production de riz pluvial, alors que l'inverse serait vrai dans le cas de l'Asie du Sud-Est, Mais au-delà de la bipolarité simplificatrice entre ces deux sous-espèces, la diversité génétique actuelle du riz est considérable, fruit de croisements entre espèces ou interne à $O$. sativa par sélection naturelle ou conduite par l'homme (notamment en stations depuis plus d'un siècle en Chine, au Japon et en Inde). On estime à 150.000 le nombre total de variétés cultivées, et la banque

${ }^{3}$ Pour plus de détails sur la structure complexe de l'espèce Oryza sativa, voir notamment B. Courtois, 2007. Une brève histoire du riz et de son amélioration génétique. Cirad, France. 13p. disponible sur l'archive ouverte : http://tel.archives-ouvertes.fr/tel-00376169/fr/ 
de gènes de l'Institut international de recherche sur le riz (IRRI) à Los Baños au sud de Luzon aux Philippines recèle 107.000 types différents, dont 5.000 provenant d’espèces sauvages.

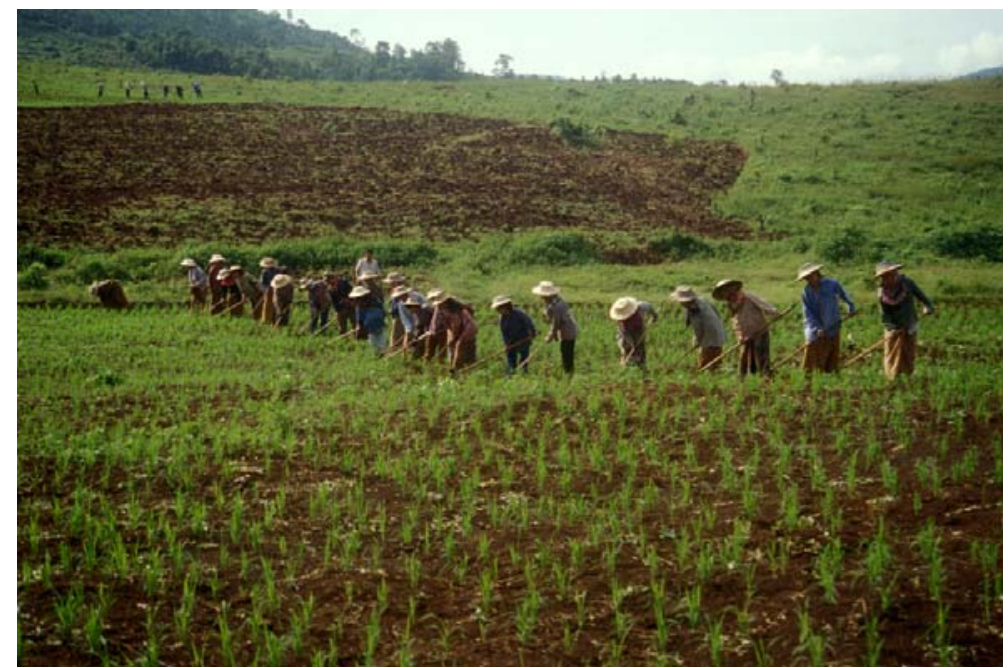

Riz pluvial - Sarclage manuel un mois après semis en poquets au Laos (@ Juillet 1985 / G. Trébuil)

Choisie comme plante modèle en génétique végétale au niveau international à cause de son relativement petit génome complètement séquencé depuis 2005, c'est à partir de ce vaste réservoir de diversité naturelle que les nouvelles techniques de sélection variétale issues de la biologie moléculaire tentent notamment de sélectionner des riz plus tolérants au déficit hydrique, à la submersion profonde, ou à l'excès de sel. Si un tel matériel végétal pouvait être créé et largement adopté dans l'écosystème à riz inondé, où des poches de pauvreté majeures demeurent notamment en Asie du Sud, il contribuerait à gagner le pari d'une augmentation de 40\% de la production rizicole d'ici 2030 afin de ne pas perdre la poursuite entamée avec la courbe démographique il y a un demi siècle et satisfaire à terme les besoins alimentaires de 4 milliards de consommateurs au niveau actuel, que l'on sait toutefois insatisfaisant en bon nombre d'endroits défavorisés. De la réussite de ce pari dépendra la stabilité politique de nombre d'états car "sans assez de grains, c'est le chaos » disait Deng Xiaoping. Au-delà des volumes produits, leur stabilité d'année en année devra donc être assurée en accordant plus d'attention aux moyens de renforcer la capacité des systèmes rizicoles à atténuer et à se remettre de chocs, soit des stress d'origine climatique (sécheresse, inondations soudaines), soit des crises économiques (cf. le triplement du prix du riz en quelques mois en 2008), les deux pouvant parfois combiner leurs effets la même année. Pour relever ce défi, le très profond ancrage social des rizicultures, ainsi que leurs structures toujours largement dominées par la petite exploitation familiale flexible, très adaptative et souvent innovante, constituent deux atouts de poids. 